\title{
Clinical Events Dictionary Derived Term
}

National Cancer Institute

\section{Source}

National Cancer Institute. Clinical Events Dictionary Derived Term. NCI Thesaurus. Code C87846.

The name of a clinical event as described in a dictionary. 